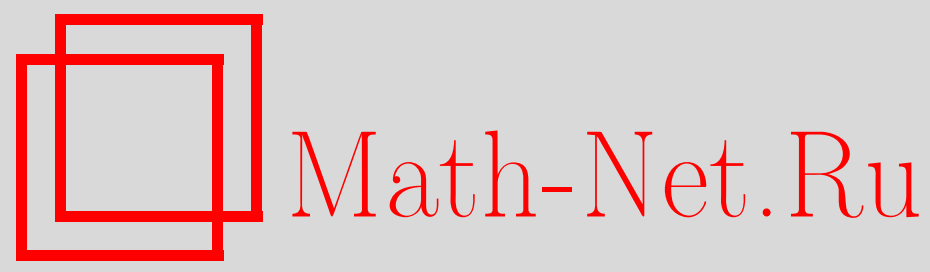

В. П. Маслов, Нелинейное среднее в экономике, Матем. заметки, 2005, том 78, выпуск 3, 377-395

DOI: https://doi.org/10.4213/mzm2595

Использование Общероссийского математического портала Math-Net.Ru подразумевает, что вы прочитали и согласны с пользовательским соглашением http://www.mathnet.ru/rus/agreement

Параметры загрузки:

IP : 18.208.226.222

26 апреля 2023 г., 14:33:51

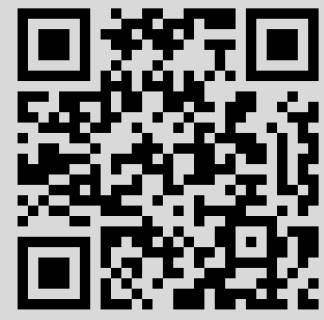


УДК 519

\title{
НЕЛИНЕЙНОЕ СРЕДНЕЕ В ЭКОНОМИКЕ
}

\author{
В.П. Маслов
}

Нелинейное осреднение по Колмогорову дополнено некоторой естественной аксиомой. Для этого осреднения доказана теорема о больших уклонениях, а также установлена связь с туннельным каноническим оператором.

Библиограффия: 17 названий.

1. Нелинейное осреднение по Колмогорову. Последовательность функций $M_{n}$ определяет регулярный тип среднего, если удовлетворяются следуюшие условия (Колмогоров):

I. $M\left(x_{1}, x_{2}, \ldots, x_{n}\right)$ непрерывна и монотонна по каждому переменному; для определенности будем считать, что $M$ возрастает по каждому переменному;

II. $M\left(x_{1}, x_{2}, \ldots, x_{n}\right)$ - симметричная функция ${ }^{1}$;

III. среднее от одинаковых чисел равно их общему значению: $M(x, x, \ldots, x)=x$;

IV. можно заменить некоторую группу значений их собственным средним, не меняя общего среднего:

$$
M\left(x_{1}, \ldots, x_{m}, y_{1}, \ldots, y_{n}\right)=M_{n+m}\left(x, \ldots, x, y_{1}, \ldots, y_{n}\right),
$$

где $x=M\left(x_{1}, \ldots, x_{m}\right)$.

Tеорема 1 (Колмогоров). При выполнении условий I-IV среднее $M\left(x_{1}, \ldots, x_{n}\right)$ имеет вид

$$
M\left(x_{1}, x_{2}, \ldots, x_{n}\right)=\psi\left(\frac{\varphi\left(x_{1}\right)+\varphi\left(x_{2}\right)+\cdots+\varphi\left(x_{n}\right)}{n}\right),
$$

где $\varphi$ - непрерывная строго монотонная функиия, а $\psi$ - обратная $к$ ней.

Доказательство теоремы см. в работе [1].

2. Основная аксиома осреднения. Для устоявшейся системы довольно очевидно, что должна быть выполнена следующая аксиома:

V. если к каждому $x_{k}$ прибавить одну и ту же величину $\omega$, то среднее увеличится на ту же величину $\omega$.

\footnotetext{
${ }^{1} \mathrm{~B}$ нашем случае симметрия следует из бозе-статистики денежных купюр.
} 
Эту аксиому я обсуждал подробно с экономистом-практиком В. Н. Батуриным.

Очевидно, что нелинейное среднее от $x_{i}$ в нормальных условиях также должно увеличиться на эту величину. Мы примем этот факт как аксиому $\mathrm{V}$.

Эта аксиома приводит к однозначному решению в нелинейном случае, т.е. ей удовлетворяет естественно линейньй случай (арифметическое среднее) и единственная с точностью до одной и той же константы, на которую мы можем умножить все доходы $x_{i}$, нелинейная функция.

На самом деле, доходы $x_{i}$ исчисляются в какой-либовалюте и, вообще говоря, должны умножаться на некоторую величину $\beta$, которая отвечает покупательной способности этой валюты, так что указанная константа (параметр $\beta$ ) изначально должна входить в определение дохода. А следовательно, мы утверждаем, что существует единственная нелинейная функция, которая удовлетворяет аксиоме V.

Функция $\varphi(x)$ имеет вид

$$
\varphi(x)=C \exp (D x)+B,
$$

где $C, D \neq 0, B$ - числа, не зависящие от $x$.

3. Полукольцо: пример линейного самосопряженного оператора. Рассмотрим полукольцо, порожденное нелинейным средним, и пространство $L_{2}$ со значениями в этом полукольце.

Вначале рассмотрим уравнение теплопроводности вида

$$
\frac{\partial u}{\partial t}=\frac{h}{2} \frac{\partial^{2} u}{\partial x^{2}}
$$

Здесь $h$ - мальй параметр, малость которого мы пока не будем использовать.

Уравнение (3) линейно. Это означает, как известно, что если $u_{1}$ и $u_{2}$ - его решения, то линейная комбинация

$$
u=\lambda_{1} u_{1}+\lambda_{2} u_{2}
$$

является его решением. Здесь $\lambda_{1}$ и $\lambda_{2}-$ константы.

Теперь сделаем следующую замену. Положим

$$
u=e^{-M / h} .
$$

Тогда для новой неизвестной функции $M(x, t)$ получим нелинейное уравнение

$$
\frac{\partial M}{\partial t}+\frac{1}{2}\left(\frac{\partial M}{\partial x}\right)^{2}-\frac{h}{2} \frac{\partial^{2} M}{\partial x^{2}}=0 .
$$

Это известное уравнение иногда называют уравнением Бюргерса ${ }^{2}$.

Решению $u_{1}$ уравнения (3) отвечает решение $M_{1}=-h \ln u_{1}$ уравнения (6), а решению $u_{2}$ уравнения (3) отвечает решение $M_{2}=-h \ln u_{2}$ уравнения (6). Решению (4) уравнения (3) отвечает решение $M=-h \ln \left(e^{-\left(M_{1}+\mu_{1}\right) / h}+e^{-\left(M_{2}+\mu_{2}\right) / h}\right)$, где $\mu_{i}=$ $-h \ln \lambda_{i}, i=1,2$.

Отсюда следует, что уравнение (6) тоже линейно, но линейно в пространстве функций, где введены следующие операции:

операция суммы $a \oplus b=-h \ln \left(e^{-a / h}+e^{-b / h}\right)$;

операция умножения $a \odot \lambda=a+\lambda$.

\footnotetext{
${ }^{2}$ Обычное уравнение Бюргерса получается из этого дифференцированием по $x$ и заменой $v=$ $\partial M / \partial x$.
} 
При этом замена $M=-h \ln u$ переводит нуль в бесконечность, а единицу - в нуль. Таким образом обобщенньм нулем в этом новом пространстве будет $\infty: \mathbf{0}=\infty$, а обобщенной единицей будет обычньй нуль: $\mathbf{1}=0$. Пространство функций, в котором введены операции $\oplus$ и $\odot$, с присоединенным к пространству нулем $\mathbf{0}$ и единицей $\mathbf{1}$ изоморфно обычному пространству функций с обькновенным умножением и сложением.

Можно себе представить, таким образом, что где-то на другой планете люди привыкли именно к вновь введенным операциям $\oplus$ и $\odot$, и с их точки зрения уравнение (6) является линейньм.

То, что здесь было описано, разумеется, тривиально, и людям нашей планеты нет смысла переучивать арифметические операции, поскольку можно заменой функции перейти от уравнения (6) к уравнению (3), линейному в привычном для нас смысле. Однако “королевство кривых зеркал", которое дает это полукольцо, оказьвается, связано с “капиталистической" экономикой [2].

В пространстве функций со значениями в кольце $a \oplus b=-h \ln \left(e^{-a / h}+e^{-b / h}\right)$, $\lambda \odot b=\lambda+b$ введем скалярное произведение

$$
\left(M_{1}, M_{2}\right)=-h \ln \int e^{\left(M_{1}+W_{2}\right) / h} d x
$$

Покажем, что оно обладает в этом пространстве билинейными свойствами, а именно:

$$
(a \oplus b, c)=(a, c) \oplus(b, c), \quad(\lambda \odot a, c)=\lambda \odot(a, c) .
$$

Действительно,

$$
\begin{aligned}
(a \oplus b, c) & =-h \ln \left(\int \exp \left(\frac{-\left(-h \ln \left(e^{-a / h}+e^{-b / h}\right)+c\right)}{h}\right) d x\right) \\
& =-h \ln \left(\int\left(e^{-a / h}+e^{-b / h}\right) e^{-c / h} d x\right) \\
& =-h \ln \left(\int e^{-(a+c) / h} d x+\int e^{-(b+c) / h} d x\right)=(a, c) \oplus(b, c), \\
(\lambda \odot a, c) & =-h \ln \int e^{-(a+\lambda) / h} e^{-c / h} d x \\
& =-h \ln \left(e^{-\lambda / h} \int e^{-(a+c) / h} d x\right)=\lambda+\ln \int e^{-(a+c) / h} d x=\lambda \odot(a, c) .
\end{aligned}
$$

Приведем пример самосопряженного оператора в этом пространстве. Рассмотрим оператор

$$
L: W \rightarrow W \odot\left(-h \ln \left(\frac{\left(W^{\prime}\right)^{2}}{h^{2}}-\frac{W^{\prime \prime}}{h}\right)\right)
$$


А теперь проверим самосопряженность:

$$
\begin{aligned}
& \left(W_{1}, L W_{2}\right)=-h \ln \int e^{-\left(W_{1}+L W_{2}\right) / h} d x \\
& \quad=-h \ln \int \exp \left[-\left(W_{1}+W_{2}-h \ln \left(\frac{\left.W_{2}^{\prime}\right)^{2}}{h^{2}}-\frac{W_{2}^{\prime \prime}}{h}\right)\right) / h\right] d x \\
& \quad=-h \ln \int e^{-W_{1} / h} e^{-W_{2} / h}\left(\frac{\left(W_{2}^{\prime}\right)^{2}}{h^{2}}-\frac{W_{2}^{\prime \prime}}{h}\right) d x=-h \ln \int e^{-W_{1} / h} \frac{d^{2}}{d x^{2}} e^{-W_{2} / h} d x \\
& =-h \ln \int \frac{d^{2}}{d x^{2}} e^{-W_{1} / h} e^{-W_{2} / h} d x=-h \ln \int e^{-W_{1} / h}\left(\frac{\left(W_{1}^{\prime}\right)^{2}}{h^{2}}-\frac{W_{1}^{\prime \prime}}{h}\right) e^{-W_{2} / h} d x \\
& \quad=-h \ln \int \exp \left[-\left(W_{1}-h \ln \left(\frac{\left(W_{1}^{\prime}\right)^{2}}{h^{2}}-\frac{W_{2}^{\prime}}{h}\right)\right) / h\right] d x \\
& =-h \ln \int e^{-\left(L W_{1}+W_{2}\right) / h} d x=\left(L W_{1}, W_{2}\right) .
\end{aligned}
$$

Также легко проверить линейность.

Построим разрешающий оператор уравнения Бюргерса: $L: W_{0} \rightarrow W$, где $W$ - решение уравнения (6), удовлетворяющее начальному условию $\left.W\right|_{t=0}=0$.

Решение уравнения (3), удовлетворяющее условию $\left.u\right|_{t=0}=u_{0}$, имеет вид

$$
u=\frac{1}{\sqrt{2 \pi h}} \int e^{-(x-\xi)^{2} /(2 t h)} u_{0}(\xi) d \xi .
$$

Учитьвая, что $u=e^{-W / h}, W=-h \ln u$, получаем разрешающий оператор $L_{t}$ уравнения Бюргерса

$$
L_{t} W_{0}=-\frac{h}{\sqrt{2 \pi h}} \ln \int e^{-\left((x-\xi)^{2} /(2 t h)+M(\xi) / h\right)} d \xi .
$$

Оператор $L_{t}$ самосопряжен в новом скалярном произведении.

4. Теорема о нелинейном среднем. Рассмотрим набор цен $\lambda_{i}$, где $i=1, \ldots, n$, и набор чисел $g_{i}$, равных числу финансовых инструментов $(\Phi И)$ - акций, облигаций и т.п. разного типа, имеющих цену $\lambda_{i}$. Цены $\lambda_{i}$ по смыслу являются положительными величинами; кроме того, без ограничения общности будем считать, что цены пронумерованы так, что для них вьполняются неравенства

$$
0<\lambda_{1}<\lambda_{i}<\lambda_{n} \quad \text { для всех } i=2, \ldots, n-1 .
$$

Полное число разного типа $Ф И$ обозначим через $G$. Это число выражается формулой

$$
G=\sum_{i=1}^{n} g_{i}
$$

Обозначим число $\Phi И$, купленных по цене $\lambda_{i}$, через $k_{i}$. Учитывая, что по цене $\lambda_{i}$ продается $g_{i}$ разных $\Phi И$, получим, что количество разных способов совершить покупку $k_{i} \Phi И$ по цене $\lambda_{i}$ выражается формулой

$$
\gamma_{i}\left(k_{i}\right)=\frac{\left(k_{i}+g_{i}-1\right) !}{k_{i} !\left(g_{i}-1\right) !} .
$$


Количество разных способов купить набор $\Phi$ И $\{k\}$, состоящий из $k_{1}, \ldots, k_{n} \Phi И$, купленных по соответствующим ценам $\lambda_{1}, \ldots, \lambda_{n}$, выражается с помощью (12) и равно

$$
\gamma(\{k\})=\prod_{i=1}^{n} \gamma_{i}\left(k_{i}\right)=\prod_{i=1}^{n} \frac{\left(k_{i}+g_{i}-1\right) !}{k_{i} !\left(g_{i}-1\right) !} .
$$

Расход денег покупателя при покупке набора $\Phi$ $\{k\}$ равен

$$
\mathscr{B}(\{k\})=\sum_{i=1}^{n} \lambda_{i} k_{i} .
$$

Произведем нелинейное осреднение расхода (14) по BR (budget restraint):

$$
M(\beta, N)=-\frac{1}{\beta N} \ln \left(\frac{N !(G-1) !}{(N+G-1) !} \sum_{\left\{k_{i}=N\right\}} \gamma(\{k\}) \exp (-\beta \mathscr{B}(\{k\}))\right),
$$

где $\beta$ - положительньй параметр.

Разобьем произвольным образом $\Phi И$ на $m$ непересекающихся групп, $m \leqslant n$. Это означает, что возьмем каким-либо способом две последовательности $i_{\alpha}$ и $j_{\alpha}$, где $\alpha=$ $1, \ldots, m$, удовлетворяюшие условиям

$$
i_{\alpha} \leqslant j_{\alpha}, \quad i_{\alpha+1}=j_{\alpha}+1, \quad \alpha=1, \ldots, m, \quad i_{1}=1, j_{m}=n,
$$

и будем считать, что $\Phi$ попадает в групп с номером $\alpha$, если номер $i$ его цены $\lambda_{i}$ удовлетворяет условию $i_{\alpha} \leqslant i \leqslant j_{\alpha}$. Заметим, что выбрать последовательности $i_{\alpha}$ и $j_{\alpha}$, удовлетворяющие условиям (16), можно множеством способов.

Число $\Phi И$, попадаюших в группу с номером $\alpha$ выражается формулой

$$
G_{\alpha}=\sum_{i=i_{\alpha}}^{j_{\alpha}} g_{i}
$$

а число $\Phi И$, купленных из группы с номером $\alpha$ при покупке набора $\{k\}=k_{1}, \ldots, k_{n}$, выражается формулой

$$
N_{\alpha}=\sum_{i=i_{\alpha}}^{j_{\alpha}} k_{i} .
$$

Набор $\{k\}$ удовлетворяет условию (31); следовательно, набор чисел $N_{\alpha}$ удовлетворяет равенству

$$
\sum_{\alpha=1}^{m} N_{\alpha}=N
$$

а также из (11) следует, что для $G_{\alpha}$ вьполняется равенство

$$
\sum_{\alpha=1}^{m} G_{\alpha}=G
$$


При этом $G$ зависит от $N$ так, что выполняется равенство

$$
\lim _{N \rightarrow \infty} \frac{G}{N}=\widetilde{g}>0
$$

Кроме того, будем считать, что разбиение $\Phi И$ на групшы (16) удовлетворяет следующему условию: $m$ не зависит от $N$, a $G_{\alpha}$ зависят от $N$ так, что выполняются равенства

$$
\lim _{N \rightarrow \infty} \frac{G_{\alpha}}{N}=\widetilde{g}_{\alpha}>0 \quad \text { для всех } \quad \alpha=1, \ldots, m
$$

Отсюда следует, что

$$
\lim _{N \rightarrow \infty} \frac{N_{\alpha}}{N} \approx \bar{n}_{\alpha}>0, \quad \sum \bar{n}_{\alpha}=1 .
$$

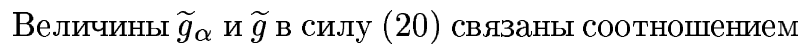

$$
\sum_{\alpha=1}^{m} \widetilde{g}_{\alpha}=\widetilde{g}
$$

Обозначим

$$
\mathcal{N}_{\alpha}(\beta, N)=\sum_{i=i_{\alpha}}^{j_{\alpha}} \frac{g_{i}}{\exp \left(\beta\left(\lambda_{i}+\nu\right)\right)-1},
$$

где $\delta>0-$ произвольньй параметр, а $\nu$ определяется из уравнения

$$
N=\sum_{i=1}^{n} \frac{g_{i}}{\exp \left(\beta\left(\lambda_{i}+\nu\right)\right)-1}
$$

Далее будем использовать обозначение

$$
\Gamma(\beta, N)=\frac{(N+G-1) !}{N !(G-1) !} \exp (-\beta N M(\beta, N))
$$

Можно рассматривать также $\beta<0$, причем при $\beta<0$ выбирается решение уравнения (25), удовлетворяющее условию $\nu<-\lambda_{n}$, а при $\beta>0$ выбирается решение, удовлетворяющее условию $\nu>-\lambda_{1}$.

Теорема 2. Пусть выполнено условие (22) и $\Delta=a N^{3 / 4+\delta}$, где а и $\delta<1 / 3$ - не зависящие от $N$ полохительные параметры. Тогда для любого $\varepsilon>0$ не зависимо от $N$ при $N \rightarrow \infty$ выполняется соотношение

$$
\begin{aligned}
& \frac{1}{\Gamma(\beta, N)} \sum_{\sum\left\{k_{i}\right\}=N, \sum_{\alpha=1}^{m}\left(N_{\alpha}(\{k\})-\mathcal{N}_{\alpha}(\beta, N)\right)^{2} \geqslant \Delta} \gamma(\{k\}) \exp (-\beta \mathscr{B}(\{k\})) \\
& \quad=O\left(\exp \left(-\frac{(1-\varepsilon) a^{2} N^{1 / 2+2 \delta}}{2 \widetilde{g} d}\right)\right)
\end{aligned}
$$


əде d определяется формулой

$$
d= \begin{cases}\frac{\exp \left(-\beta\left(\lambda_{1}+\nu\right)\right)}{\left(\exp \left(-\beta\left(\lambda_{1}+\nu\right)\right)-1\right)^{2}} & \text { при } \beta<0, \\ \frac{\exp \left(-\beta\left(\lambda_{n}+\nu\right)\right)}{\left(\exp \left(-\beta\left(\lambda_{n}+\nu\right)\right)-1\right)^{2}} & \text { при } \beta>0 .\end{cases}
$$

То есть вклад в средний расход покупателя от числа купленных $Ф И N_{\alpha}$ (порядка $N)$, которое отличается от $\mathscr{N}_{\alpha}(\beta, N)$ на величину порядка $O\left(N^{3 / 4+\delta}\right)$, является величиной әкспоненииально малой.

Таким образом, в случае когда $Ф И$ разбиваются на достаточно большие группы (условие (22)), для данного нелинейного среднего расхода покупателя число купленных $\Phi И$ грушы $\alpha$ в большинстве случаев равно приблизительно $\mathscr{N}_{\alpha}(\beta, N)$, что является аналогом закона больших чисел.

Для определения $\beta$ предлагается следующий способ. Пусть задан набор фактических покупок $N_{\alpha}^{f}$ группы финансовых инструментов $\alpha=1,2, \cdots, m$. Рассмотрим следующую функцию от $\beta$ :

$$
D N(\beta)=\sum_{\alpha=1}^{m}\left(N_{\alpha}^{f}-\mathscr{N}_{\alpha}\left(\beta, \nu_{\alpha}\right)\right)^{2},
$$

где $\mathscr{N}_{\alpha}\left(\beta, \nu_{\alpha}\right)$ выражается по формуле (24). В качестве $\beta$ выбирается значение, при котором функция (29) минимальна.

Минимум этой величины, которая может быть определена, например, на фондовой бирже, показывает степень случайности разложения $\Phi$ Ио портфелям трейдеров. Если минимум равен нулю, то распределение по портфелям случайно (см. [3]).

Прежде чем приступить к доказательству, докажем несколько утверждений и лемм.

Предположим, что покупатель располагает суммой денег $B$ (budget restraint), но при этом не обязательно потратит всю эту сумму на покупки, и предположим, что он покупает $N \Phi$ И. Полное число разных вариантов покупок, при которых покупается $N \Phi$ и и тратится сумма, не превышающая $B$, выражается формулой [4]

$$
\sigma(B, N)=\sum_{\{k=N\}} \gamma(\{k\}) \Theta(B-\mathscr{B}(\{k\})) .
$$

Здесь $\mathscr{B}(\{k\}) \leqslant B, \mathscr{B}(\{k\})$ определяется формулой $(14), \Theta(x)$ - функция Хевисайда:

$$
\Theta(x)= \begin{cases}0 & \text { при } x<0, \\ 1 & \text { при } x \geqslant 1,\end{cases}
$$

a $\sum_{\{k\}}^{N}$ обозначает сумму по всем наборам $\{k\}$ целых неотрицательных чисел $k_{1}, \ldots, k_{n}$, удовлетворяюших условию

$$
\sum_{i=1}^{n} k_{i}=N .
$$

Заметим, что в силу (10) для любого набора $\{k\}$, удовлетворяющего (31), соответствуюший расход (14) удовлетворяет неравенствам

$$
0<N \lambda_{1} \leqslant \mathscr{B}(\{k\}) \leqslant N \lambda_{n}
$$


Число вариантов покупки $\sigma(B, N)(30)$ является кусочно-постоянной функцией переменной $B$ и обладает следуюшими свойствами:

$$
\begin{array}{lll}
\sigma(B, N) \leqslant \sigma\left(B^{\prime}, N\right) & \text { при } B \leqslant B^{\prime}, \\
\sigma(B, N)=0 & \text { при } B<N \lambda_{1}, \\
\sigma(B, N)=\sigma\left(N \lambda_{n}, N\right) & \text { при } B \geqslant N \lambda_{n} .
\end{array}
$$

Эти свойства $\sigma(B, N)$ следуют непосредственно из определений $(30)$ и неравенства $(32)$.

УТВЕРЖДЕНИЕ 1. Для любого положительного $\beta$ и любого $N$ выполняется тохсдество

$$
\int_{0}^{\infty} e^{-\beta B} d \sigma(B, N)=\Gamma(\beta, N)
$$

ДоКАЗАТЕЛЬСТво. Подставляя (30) в левую часть равенства (33) и меняя порядок суммирования и интегрирования, получим

$$
\int_{0}^{\infty} e^{-\beta B} d \sigma(B, N)=\sum_{\{k\}}^{N} \gamma(\{k\}) \int_{0}^{\infty} e^{-\beta B} d \Theta(B-\mathscr{B}(\{k\}))
$$

Теперь воспользуемся равенством

$$
\int_{0}^{\infty} e^{-\beta B} d \Theta\left(B-B_{0}\right)=e^{-\beta B_{0}}
$$

которое справедливо для любьх положительньх $\beta$ и $B_{0}$. Учитьвая $(35)$, получим из $(34)$ следующее равенство:

$$
\int_{0}^{\infty} e^{-\beta B} d \sigma(B, N)=\sum_{\{k\}}^{N} \gamma(\{k\}) \exp (-\beta \mathscr{B}(\{k\}))
$$

Из (15) и (26) следует, что выражение в правой части равенства $(36)$ равно $\Gamma(\beta, N)$. Таким образом, утверждение доказано.

Введем следующую функцию $\zeta(\beta, \nu)$, определенную для всех $\nu>-\lambda_{1}$ и $\beta>0$ :

$$
\zeta(\beta, \nu)=\sum_{N=0}^{\infty} \Gamma(\beta, N) e^{-\beta N \nu}
$$

УТВЕРЖДЕНИЕ 2. Для любых $\nu>-\lambda_{1} u \beta>0$ справедливо равенство

$$
\zeta(\beta, \nu)=\prod_{i=1}^{n} \frac{1}{\left(1-\exp \left(-\beta\left(\lambda_{i}+\nu\right)\right)\right)^{g_{i}}}
$$


ДоКАЗАТЕЛЬСТВо. Согласно формулам (15), (26) имеем

$$
\sum_{N=0}^{\infty} \Gamma(\beta, N) e^{-\beta N \nu}=\sum_{N=0}^{\infty} e^{-\beta N \nu} \sum_{\{k\}}^{N} \gamma(\{k\}) \exp (-\beta \mathscr{B}(\{k\}))
$$

Учитьвая явньй вид $\gamma(\{k\})(13)$ и $\mathscr{B}(\{k\})(14)$, получим, что сумма в равенстве (39) распадается на произведение сумм:

$$
\sum_{N=0}^{\infty} e^{-\beta N \nu} \sum_{\{k\}}^{N} \gamma(\{k\}) \exp (-\beta \mathscr{B}(\{k\}))=\prod_{i=1}\left(\sum_{k_{i}=1}^{\infty} \frac{\left(k_{i}+g_{i}-1\right) !}{k_{i} !\left(g_{i}-1\right) !} e^{-\beta\left(\lambda_{i}+\nu\right) k_{i}}\right) .
$$

Воспользуемся теперь равенством

$$
\sum_{k=0}^{\infty} \frac{(k+g-1) !}{k !(g-1) !} x^{k}=\frac{1}{(1-x)^{g}}
$$

справедливьм для любого натурального $g$ и любого $x$ такого, что $|x|<1$. Так как $\nu>-\lambda_{1}$ и $\beta>0$, в силу (41) из (37), (39), (40) следует (38). Утверждение доказано.

Для доказательства некоторых свойств функции $\Gamma(\beta, N)($ см. $(26))$ используется следующая лемма.

Лемма 1. Функиии $z_{n}\left(x_{1}, \ldots, x_{n} ; N\right)$, имеющие вид

$$
z_{l}\left(x_{1}, \ldots, x_{l} ; N\right)=\sum_{\{k\}}^{N} \exp \left(\sum_{i=1}^{l} x_{i} k_{i}\right)
$$

при произвольных иелых $l u N$, удовлетворяюших условиям $l \geqslant 2 u N \geqslant 1, u n p u$ всех действительных $x_{1}, \ldots, x_{n}$, удовлетворяют неравенству

$$
z_{l}\left(x_{1}, \ldots, x_{l}, N\right)^{2}>z_{l}\left(x_{1}, \ldots, x_{l}, N-1\right) z_{l}\left(x_{1}, \ldots, x_{l}, N+1\right) .
$$

ДоказАтЕльство. Докажем утверждение леммы по индукции. Сначала докажем, что (43) справедливо при $l=2$. Если $l=2$ и $x_{2}=x_{1}$, то при всех целых неотрицательных $N$ функция (42) равна

$$
z_{2}\left(x_{1}, x_{1} ; N\right)=\sum_{k=0}^{N} e^{x_{1} N}=(N+1) e^{x_{1} N}
$$

и выполнение (43) в этом случае проверяется элементарно. Если $l=2$ и $x_{2} \neq x_{1}$, то для всех целых неотрицательных $N$ функция (42) имеет вид

$$
z_{2}\left(x_{1}, x_{2} ; N\right)=\sum_{k=0}^{N} e^{x_{1} k+x_{2}(N-k)}=e^{x_{2} N} \frac{1-e^{\left(x_{1}-x_{2}\right)(N+1)}}{1-e^{x_{1}-x_{2}}} .
$$


Подставляя (44) в (43), получим после несложных преобразований, что выполнение неравенства (43) в этом случае эквивалентно вьполнению следующего неравенства:

$$
\frac{1}{2}\left(e^{x_{1}-x_{2}}+e^{x_{2}-x_{1}}\right)>1
$$

Однако неравенство (45) справедливо для всех не равных друг другу чисел $x_{1}$ и $x_{2}$ в силу вьпуклости функции $y(x)=\exp (x)$. Таким образом, справедливость $(43)$ для $l=2$ доказана. Предположим теперь, что неравенство (43) доказано при $l=k$, где $k \geqslant 2$, и докажем, что отсюда следует справедливость этого неравенства при $n=l+1$. Заметим, что функции (42) обладают следующим свойством, которое элементарно следует из явного вида этих функций:

$$
z_{k+1}\left(x_{1}, \ldots, x_{k+1} ; N\right)=e^{x_{k+1} N} \sum_{m=0}^{N} z_{k}\left(\widetilde{x}_{1}, \ldots, \widetilde{x}_{k} ; m\right)
$$

где $\widetilde{x}_{i}=x_{i}-x_{k+1}, i=1, \ldots, n$. Подстановка (46) в неравенство (43) после несложных преобразований приводит к тому, что выполнение неравенства (43) при $n=k+1$ эквивалентно справедливости неравенства

$$
z_{k}(\widetilde{x} ; 0) z_{k}(\widetilde{x} ; N)+\sum_{m=0}^{N-1}\left(z_{k}(\widetilde{x} ; k+1) z_{k}(\widetilde{x} ; N)-z_{k}(\widetilde{x} ; k) z(\widetilde{x} ; N+1)\right)>0
$$

где $z_{k}(\widetilde{x} ; m)$ обозначает $z_{k}\left(\widetilde{x}_{1}, \ldots, \widetilde{x}_{k} ; m\right)$. По предположению неравенство (43) справедливо при $n=k$; отсюда следует, что для всех $k=0, \ldots, N-1$ вьполняется

$$
z_{k}(\widetilde{x} ; k+1) z_{k}(\widetilde{x} ; N)-z_{k}(\widetilde{x} ; k) z(\widetilde{x} ; N+1)>0
$$

а это означает, что все слагаемые в левой части неравенства (47) положительные, т.е. неравенство (47) выполняется. Таким образом, справедливость (43) при $l=k+1$ доказана и, следовательно, утверждение леммы также доказано по индукции.

Следствием леммы 1 является вьпуклость логарифма функции $\Gamma(\beta, N)(26)$ по дискретной переменной $N$.

УТВЕРЖДЕНИЕ 3. При всех натуральных $N$ и всех положительных $\beta$ выполняются неравенства

$$
\begin{gathered}
\Gamma(\beta, N)^{2}>\Gamma(\beta, N-1) \Gamma(\beta, N+1), \\
\Gamma(\beta, N-1)<e^{\beta \lambda_{1}} \Gamma(\beta, N) .
\end{gathered}
$$


ДокАЗАТЕЛЬСтво. Для доказательства этих неравенств достаточно заметить, что $\Gamma(\beta, N)(26)$ согласно формуле (15) может быть записано в виде

$$
\Gamma(\beta, N)=\sum_{\{k\}}^{N} \gamma(\{k\}) \exp (-\beta \mathscr{B}(\{k\})) .
$$

Правая часть равенства (51) равна функции (42) при

$$
\begin{gathered}
l=G, \quad x_{1}=\cdots=x_{g_{1}}=-\beta \lambda_{1}, \quad x_{g_{1}+1}=\cdots=x_{g_{1}+g_{2}}=-\beta \lambda_{2}, \ldots, \\
x_{G-g_{n}+1}=\cdots=x_{G}=-\beta \lambda_{G}
\end{gathered}
$$

это равенство следует из формулы

$$
\sum_{0 \leqslant m_{1}, \ldots, m_{g}}^{m_{1}+\cdots+m_{g}=k} 1=\frac{(k+g-1) !}{k !(g-1) !} .
$$

Таким образом, (49) следует из (43), а для доказательства (50) достаточно воспользоваться равенством (46).

Из свойства (49) следует еще одно свойство Г $(\beta, N)$.

УТВЕРЖДЕНИЕ 4. При любом заданном положительном $\beta$ для любого неотрииательного иелого $N$ существует $\nu>-\lambda_{1}$ такое, что для всех $N^{\prime} \neq N$

$$
\Gamma(\beta, N) e^{-\beta N \nu}>\Gamma\left(\beta, N^{\prime}\right) e^{-\beta N^{\prime} \nu}
$$

ДоКАЗАТЕЛЬСТво. В силу (49) выберем любое $\nu$, удовлетворяющее неравенству

$$
\frac{1}{\beta} \ln \left(\frac{\Gamma(\beta, N+1)}{\Gamma(\beta, N)}\right)<\nu<\frac{1}{\beta} \ln \left(\frac{\Gamma(\beta, N)}{\Gamma(\beta, N-1)}\right) .
$$

Из (50) следует, что такое $\nu$ удовлетворяет неравенству $\nu>-\lambda_{1}$. Кроме того, из (49) и (54) следует выполнение неравенств

$$
\begin{array}{ll}
e^{-\beta\left(N^{\prime}+1\right) \nu} \Gamma\left(\beta, N^{\prime}+1\right)>e^{-\beta N^{\prime} \nu} \Gamma\left(\beta, N^{\prime}\right) & \text { для всех } \quad N^{\prime}, 0 \leqslant N^{\prime} \leqslant N-1, \\
e^{-\beta\left(N^{\prime}+1\right) \nu} \Gamma\left(\beta, N^{\prime}+1\right)<e^{-\beta N^{\prime} \nu} \Gamma\left(\beta, N^{\prime}\right) & \text { для всех } \quad N^{\prime}, \quad N \leqslant N^{\prime} .
\end{array}
$$

Однако, справедливость неравенств (55) означает вьполнение неравенства (53) при всех $N^{\prime} \neq N$. Утверждение доказано.

Теперь докажем еще одну лемму, которая позволит оценить нелинейное осреднение (15) с помошью равенства (37). Пусть $p_{l}, l=0,1, \ldots,-$ последовательность чисел, удовлетворяющих условиям

$$
p_{l} \geqslant 0 \quad \text { для всех } l=0,1, \ldots, \quad \sum_{l=0}^{\infty} p_{l}=1,
$$


а так же

$$
\begin{aligned}
\bar{l} & \equiv \sum_{l=0}^{\infty} l p_{l}<\infty, \\
D l & \equiv \sum_{l=0}^{\infty}(l-\bar{l})^{2} p_{l}<\infty .
\end{aligned}
$$

Из условий (56) следует, что числа $p_{l}$ ограничены сверху, и из них можно выбрать максимальное. То есть существует индекс, которьй далее обозначим через $L$, такой, что выполняется условие

$$
p_{L} \geqslant p_{l} \quad \text { для всех } l=0,1, \ldots
$$

ЛЕмма 2. Для произвольной последовательности чисел $p_{l}, l=0,1, \ldots$, удовлетворяющей условиям (56)-(59), справедливь неравенства

$$
\begin{gathered}
|L-\bar{l}| \leqslant(3 D l)^{3 / 4}, \\
p_{L} \geqslant \frac{1}{\sqrt{27 D l}} .
\end{gathered}
$$

ДокАЗАТЕЛЬСТво. Для любого $\Delta>0$ вьполняется неравенство Чебышева

$$
\sum_{l=0}^{\infty} p_{l} \Theta(|l-\bar{l}|-\Delta) \leqslant \sum_{l=0}^{\infty} p_{l} \frac{(l-\bar{l})^{2}}{\Delta^{2}}=\frac{D l}{\Delta^{2}}
$$

Из (62) и $(56),(59)$ следует, что для любого $\Delta>0$ имеет место цепочка неравенств

$$
2 \Delta p_{L} \geqslant \sum_{|l-\bar{l}|<\Delta} p_{l} \geqslant 1-\frac{D l}{\Delta^{2}}
$$

Из (63) получаем

$$
p_{L} \geqslant \frac{1}{2 \Delta}\left(1-\frac{D l}{\Delta^{2}}\right) \quad \text { для любого } \Delta>0 .
$$

Элементарное исследование на максимум по переменной $\Delta$ функции, стоящей в правой части неравенства (64), показывает, что эта функция достигает максимального значения $1 / \sqrt{27 D l}$ при $\Delta=\sqrt{3 D n}$. Неравенство (61), таким образом, доказано. Возьмем теперь $\Delta=|L-\bar{l}|$; тогда из (62) и (56) следует

$$
p_{L} \leqslant \sum_{l=0}^{\infty} p_{l} \Theta(|l-\bar{l}|-|L-\bar{l}|) \leqslant \frac{D l}{(L-\bar{l})^{2}} .
$$

Учитьвая (61), из (65) получим неравенство (60).

Каждой группе соответствует свое нелинейное осреднение расхода; аналогично (15) осреднение расхода на $\Phi И$ из группы с номером $\alpha$ выражается формулой

$$
M_{\alpha}\left(\beta, N_{\alpha}\right)=-\frac{1}{\beta N_{\alpha}} \ln \left(\frac{N_{\alpha} !\left(G_{\alpha}-1\right) !}{\left(N_{\alpha}+G_{\alpha}-1\right) !} \sum_{\{k\}_{\alpha}}^{N_{\alpha}} \prod_{i=i_{\alpha}}^{j_{\alpha}}\left(\gamma_{i}\left(k_{i}\right) e^{-\beta \lambda_{i} k_{i}}\right)\right),
$$


где $\gamma_{i}\left(k_{i}\right)$ выражается формулой $(12)$, a $\sum_{\{k\}_{\alpha}}^{N_{\alpha}}$ обозначает сумму по всем наборам $\{k\}_{\alpha}$ целых неотрицательных чисел $k_{i_{\alpha}}, \ldots, k_{j_{\alpha}}$, удовлетворяющим условию (18). Аналогично (26) и (37) для всех неотрицательных целых $N_{\alpha}$, для всех $\beta>0$ и всех $\nu>-\lambda_{1}$ определяются функции $\Gamma_{\alpha}\left(\beta, N_{\alpha}\right)$ и $\zeta_{\alpha}(\beta, \nu)$ :

$$
\begin{aligned}
\Gamma_{\alpha}\left(\beta, N_{\alpha}\right) & =\frac{\left(N_{\alpha}+G_{\alpha}-1\right) !}{N_{\alpha} !\left(G_{\alpha}-1\right) !} \exp \left(-\beta N_{\alpha} M_{\alpha}\left(\beta, N_{\alpha}\right)\right), \\
\zeta_{\alpha}(\beta, \nu) & =\sum_{N_{\alpha}=0}^{\infty} \Gamma_{\alpha}\left(\beta, N_{\alpha}\right) e^{-\beta N_{\alpha} \nu} .
\end{aligned}
$$

УТВЕРЖДЕНИЕ 5. Имеют место равенства

$$
\begin{aligned}
\Gamma(\beta, N) & =\sum_{\left\{N_{\alpha}\right\}}^{N} \prod_{\alpha=1}^{m} \Gamma_{\alpha}\left(\beta, N_{\alpha}\right), \\
\zeta(\beta, \nu) & =\prod_{\alpha=1}^{m} \zeta_{\alpha}(\beta, \nu), \\
\zeta_{\alpha}(\beta, \nu) & =\prod_{i=i_{\alpha}}^{j_{\alpha}} \frac{1}{\left(1-\exp \left(-\beta\left(\lambda_{i}+\nu\right)\right)\right)^{g_{i}}},
\end{aligned}
$$

əде $\sum_{\left\{N_{\alpha}\right\}}^{N}$ обозначает сумму по всем наборам $\left\{N_{\alpha}\right\}$ иельх неотричательньх чисел $N_{1}, \ldots, N_{m}$, удовлетворяющим условию (19).

ДокАЗАтЕльство. Равенства (70) и (71) следуют из (38). Для доказательства (69) достаточно воспользоваться формулой (51) и учесть, что для всех $\Gamma_{\alpha}\left(\beta, N_{\alpha}\right), \alpha=$ $1, \ldots, m$, имеют место аналогичные формулы, а также учесть, что в формуле (51) слагаемые суммы в силу явного вида $\gamma(\{k\})(13)$ и $\mathscr{B}(\{k\})(14)$ распадаются на произведение.

Среднее число $Ф И$, купленных из групшы с номером $\alpha$ при покупке в целом $N \Phi И$, в зависимости от $\beta$ определяется следуюшим образом:

$$
\bar{N}_{\alpha}(\beta, N)=\frac{1}{\Gamma(\beta, N)} \sum_{\{k\}}^{N} N_{\alpha}(\{k\}) \gamma(\{k\}) \exp (-\beta \mathscr{B}(\{k\})),
$$

где $N_{\alpha}(\{k\})$ зависит от набора $\{k\}$ согласно формуле (18). При рассмотрении средних (72) также нам понадобятся следующие функции:

$$
\begin{aligned}
\tilde{N}(\beta, \nu) & =\frac{1}{\zeta(\beta, \nu)} \sum_{N=0}^{\infty} N \Gamma(\beta, N) e^{-\beta N \nu}, \\
D \tilde{N}(\beta, \nu) & =\frac{1}{\zeta(\beta, \nu)} \sum_{N=0}^{\infty}(N-\bar{N}(\beta, \nu))^{2} \Gamma(\beta, N) e^{-\beta N \nu}, \\
\tilde{N}_{\alpha}(\beta, \nu) & =\frac{1}{\zeta(\beta, \nu)} \sum_{\{k\}} N_{\alpha}(\{k\}) \gamma(\{k\}) \exp (-\beta(\mathscr{B}(\{k\})+\nu N(\{k\}))), \\
D \tilde{N}_{\alpha}(\beta, \nu) & =\frac{1}{\zeta(\beta, \nu)} \sum_{\{k\}}\left(N_{\alpha}(\{k\})-\tilde{N}_{\alpha}(\beta, \nu)\right)^{2} \gamma(\{k\}) \exp (-\beta(\mathscr{B}(\{k\})+\nu N(\{k\}))),
\end{aligned}
$$

где $N(\{k\})$ зависит от набора $\{k\}$ по формуле $(31)$, а $\sum_{\{k\}}$ обозначает сумму по всем наборам $\{k\}$ целых неотрищательных чисел $k_{1}, \ldots, k_{n}$. 
УТВЕРЖДЕНИЕ 6. Для величин (73)-(76) имеют место тождества

$$
\begin{aligned}
\tilde{N}(\beta, \nu) & =\sum_{i=1}^{n} \frac{g_{i}}{\exp \left(\beta\left(\lambda_{i}+\nu\right)\right)-1}, \\
D \widetilde{N}(\beta, \nu) & =\sum_{i=1}^{n} \frac{g_{i} \exp \left(\beta\left(\lambda_{i}+\nu\right)\right)}{\left(\exp \left(\beta\left(\lambda_{i}+\nu\right)\right)-1\right)^{2}}, \\
\tilde{N}_{\alpha}(\beta, \nu) & =\sum_{i=i_{\alpha}}^{j_{\alpha}} \frac{g_{i}}{\exp \left(\beta\left(\lambda_{i}+\nu\right)\right)-1}, \\
D \widetilde{N}_{\alpha}(\beta, \nu) & =\sum_{i=i_{\alpha}}^{j_{\alpha}} \frac{g_{i} \exp \left(\beta\left(\lambda_{i}+\nu\right)\right)}{\left(\exp \left(\beta\left(\lambda_{i}+\nu\right)\right)-1\right)^{2}} .
\end{aligned}
$$

ДокАЗАТЕльСтво. Сначала заметим, что величины (73) и (74) можно записать в виде

$$
\begin{aligned}
\widetilde{N}(\beta, \nu) & =-\frac{1}{\beta} \frac{\partial}{\partial \nu} \ln (\zeta(\beta, \nu)), \\
D \widetilde{N}(\beta, \nu) & =-\frac{1}{\beta} \frac{\partial}{\partial \nu} \tilde{N}(\beta, \nu),
\end{aligned}
$$

в чем можно убедиться дифференцированием $\zeta(\beta, \nu)(37)$. После чего, подставляя (38) в (81) и (82), получим (77) и (78). Теперь, учитьвая явньй вид $\gamma(\{k\})(13)$ и $\mathscr{B}(\{k\})(14)$, а также формулы $(66),(67)$ и $(70)$, запишем выражения $(75),(76)$ в виде, аналогичном (73), (74):

$$
\begin{aligned}
& \widetilde{N}_{\alpha}(\beta, \nu)=\frac{1}{\zeta_{\alpha}(\beta, \nu)} \sum_{N_{\alpha}=0}^{\infty} N_{\alpha} \Gamma_{\alpha}\left(\beta, N_{\alpha}\right) e^{-\beta N_{\alpha} \nu} \\
& D \widetilde{N}(\beta, \nu)=\frac{1}{\zeta_{\alpha}(\beta, \nu)} \sum_{N_{\alpha}=0}^{\infty}\left(N_{\alpha}-\bar{N}_{\alpha}(\beta, \nu)\right)^{2} \Gamma_{\alpha}\left(\beta, N_{\alpha}\right) e^{-\beta N_{\alpha} \nu}
\end{aligned}
$$

Следовательно, для величин (75) и (76) имеют место формулы, аналогичные формулам $(81)$ и $(82)$, только $\zeta(\beta, \nu)$ надо заменить на $\zeta_{\alpha}(\beta, \nu) ;$ подставляя в эти формулы $(71)$, получим (79) и (80). Утверждение доказано.

Для доказательства экспоненциальной оценки в утверждении теоремы 2 используется следующее

УТВеРЖДЕНИЕ 7. Для всех положительных $\Delta$ выполняется неравенство

$$
\begin{aligned}
& \frac{1}{\zeta(\beta, \nu)} \sum_{\{k\}} \Theta\left(\sum_{\alpha=1}^{m}\left|N_{\alpha}-\widetilde{N}_{\alpha}(\beta, \nu)\right|-\Delta\right) \gamma(\{k\}) \exp (\beta(\mathscr{B}(\{k\})+\nu N(\{k\}))) \\
& \quad \leqslant 2^{m} \exp \left(-\frac{\Delta^{2}}{2 G d}\right)
\end{aligned}
$$

где d определяется формулой (28). 
ДоКАЗАТЕЛЬСТво. Воспользуемся следующими свойствами функции гиперболического косинуса $\cosh (x)=\left(e^{x}+e^{-x}\right) / 2$ :

$$
\prod_{\alpha=1}^{m} \cosh \left(x_{\alpha}\right) \geqslant\left(\cosh \left(\frac{\Delta}{m}\right)\right)^{m} \forall x_{\alpha}: \sum_{\alpha=1}^{m}\left|x_{\alpha}\right| \geqslant \Delta, \quad \frac{1}{\cosh (x)} \leqslant 2 e^{-x} .
$$

Неравенства (86) позволяют написать с помощью формулы (69) следуюшее (экспоненциальное) неравенство Чебышева:

$$
\begin{array}{r}
\sum_{\{k\}} \Theta\left(\sum_{\alpha=1}^{m}\left|N_{\alpha}-\widetilde{N}_{\alpha}(\beta, \nu)\right|-\Delta\right) \gamma(\{k\}) \exp (\beta(\mathscr{B}(\{k\})+\nu N(\{k\}))) \\
\leqslant 2^{m} e^{-c \Delta} \prod_{\alpha=1}^{m}\left(\sum_{N_{\alpha}=0}^{\infty} \Gamma_{\alpha}\left(\beta, N_{\alpha}\right) e^{\beta \nu N_{\alpha}} \cosh \left(c\left(N_{\alpha}-\widetilde{N}_{\alpha}(\beta, \nu)\right)\right)\right),
\end{array}
$$

где $c$-произвольноеположительное число. Учитьвая теперь (68) и (70), перепишем (87) в виде

$$
\begin{gathered}
\frac{1}{\zeta(\beta, \nu)} \sum_{\{k\}} \Theta\left(\sum_{\alpha=1}^{m}\left|N_{\alpha}-\widetilde{N}_{\alpha}(\beta, \nu)\right|-\Delta\right) \gamma(\{k\}) \exp (\beta(\mathscr{B}(\{k\})+\nu N(\{k\}))) \\
\leqslant 2^{m} e^{-c \Delta} \prod_{\alpha=1}^{m} \frac{1}{2 \zeta_{\alpha}(\beta, \nu)}\left(\exp \left(-c \widetilde{N}_{\alpha}(\beta, \nu)\right) \zeta_{\alpha}\left(\beta, \nu+\frac{c}{\beta}\right)\right. \\
\left.+\exp \left(c \widetilde{N}_{\alpha}(\beta, \nu)\right) \zeta_{\alpha}\left(\beta, \nu-\frac{c}{\beta}\right)\right) .
\end{gathered}
$$

Далее используем тождество

$$
\begin{gathered}
\ln \left(\zeta_{\alpha}\left(\beta, \nu+\frac{c}{\beta}\right)\right)=\ln \left(\zeta_{\alpha}(\beta, \nu)\right)+\frac{c}{\beta} \frac{\partial}{\partial \nu} \ln \left(\zeta_{\alpha}(\beta, \nu)\right) \\
+\int_{\nu}^{\nu+c / \beta} d \nu^{\prime}\left(\nu+\frac{c}{\beta}-\nu^{\prime}\right) \frac{\partial^{2}}{\partial \nu^{\prime 2}} \ln \left(\zeta_{\alpha}\left(\beta, \nu^{\prime}\right)\right)
\end{gathered}
$$

справедливое в силу двукратной дифференцируемости функции (71) по переменной $\nu$. В силу явного вида функции $\zeta_{\alpha}(\beta, \nu)(71)$ имеем

$$
\frac{\partial^{2}}{\partial \nu^{2}} \ln \left(\zeta_{\alpha}(\beta, \nu)\right)=\beta^{2} \sum_{i=i_{\alpha}}^{j_{\alpha}} \frac{g_{i} \exp \left(-\beta\left(\lambda_{i}+\nu\right)\right)}{\left(\exp \left(-\beta\left(\lambda_{i}+\nu\right)\right)-1\right)^{2}} \leqslant \beta^{2} G_{\alpha} d
$$

где $d$ выражается формулой (28). Используя неравенство (90) и формулы (79), (81), получим из (89) следующее неравенство:

$$
-c \widetilde{N}_{\alpha}(\beta, \nu)+\ln \left(\zeta_{\alpha}\left(\beta, \nu+\frac{c}{\beta}\right)\right)-\ln \left(\zeta_{\alpha}(\beta, \nu)\right) \leqslant \frac{G_{\alpha}}{2} d c^{2} .
$$


В силу (91) и (20) из (88) следует неравенство

$$
\begin{aligned}
& \frac{1}{\zeta(\beta, \nu)} \sum_{\{k\}} \Theta\left(\sum_{\alpha=1}^{m}\left|N_{\alpha}-\tilde{N}_{\alpha}(\beta, \nu)\right|-\Delta\right) \gamma(\{k\}) \exp (\beta(\mathscr{B}(\{k\})+\nu N(\{k\}))) \\
& \leqslant 2^{m} \exp \left(-c \Delta+\frac{G d c^{2}}{2}\right) .
\end{aligned}
$$

Выражение в правой части неравенства (92) содержит произвольньй положительный параметр $c$; минимальное значение этого выражения достигается при $c=\Delta / d$, и оно равно $2^{m} \exp \left(-\Delta^{2} / 2 d\right)$, а значит, (85) следует из (92). Утверждение доказано.

Будем считать, что $\lambda_{1}$ и $\lambda_{n}$ не зависят от $N$; согласно (10) это означает, что цены $\lambda_{i}$, $i=1, \ldots, n$, лежат в независящем от $N$ диапазоне. Характер зависимости $n$ от $N$ никак ограничивать не будем, на полученных результатах эта зависимость не сказывается; заметим только, что согласно (11) для $n$ имеет место неравенство $n \leqslant G$. Оговоренные условия допускают большой произвол в выборе $\lambda_{i}$ и $g_{i}$, а также в разбиении $\Phi И$ на группы. В связи с этим написать асимптотику нелинейных осреднений (15) и (66) и средних (72) в пределе при $N \rightarrow \infty$ в общем случае невозможно; однако оказьвается, что можно доказать некоторые свойства этих величин в таком предельном случае.

ДоКАЗАТЕЛЬСТво ТЕОРЕМЫ 2. Согласно утверждению 4 выберем для заданных $\beta$ и $N$ такое $\nu^{\prime}$, что для него выполняется неравенство (53). В силу формул $(37),(38)$ и (73), (74), (77), (78) последовательность

$$
p_{l}=\frac{\Gamma(\beta, l) e^{\beta l \nu^{\prime}}}{\zeta\left(\beta, \nu^{\prime}\right)}
$$

является последовательностью, для которой применима лемма 2. Это означает, что для заданных $\beta, N$ и соответствующего им $\nu^{\prime}$ вьполняются неравенства

$$
\begin{gathered}
\left|\tilde{N}\left(\beta, \nu^{\prime}\right)-N\right| \leqslant\left(3 D \tilde{N}\left(\beta, \nu^{\prime}\right)\right)^{3 / 4} \\
1>\frac{\Gamma(\beta, N) e^{\beta N \nu^{\prime}}}{\zeta\left(\beta, \nu^{\prime}\right)} \geqslant \frac{1}{\sqrt{27 D \tilde{N}\left(\beta, \nu^{\prime}\right)}} .
\end{gathered}
$$

Заметим теперь, что функция $\widetilde{N}(\beta, \nu)(77)$ обладает следуюшими свойствами при всех $\nu>-\lambda_{1}$, если $\beta<0$, и всех $\nu<-\lambda_{n}$, если $\beta>0$ :

$$
\begin{aligned}
\tilde{N}(\beta, \nu) \geqslant 0, & \lim _{\nu \rightarrow \infty} \tilde{N}(\beta, \nu)=0, \quad \lim _{\nu \rightarrow-\lambda_{1}+0} \tilde{N}(\beta, \nu)=\lim _{\nu \rightarrow-\lambda_{n}-0} \tilde{N}(\beta, \nu)=+\infty, \\
& \frac{1}{\beta} \frac{\partial \widetilde{N}}{\partial \nu}(\beta, \nu)=\sum_{i=1}^{n} \frac{g_{i} \exp \left(-\beta\left(\lambda_{i}+\nu\right)\right)}{\left(\exp \left(-\beta\left(\lambda_{i}+\nu\right)\right)-1\right)^{2}}>0, \\
& \left|\frac{\partial \tilde{N}}{\partial \nu}(\beta, \nu)\right| \leqslant \begin{cases}-\frac{\beta G \exp \left(-\beta\left(\lambda_{1}+\nu\right)\right)}{\left(\exp \left(-\beta\left(\lambda_{1}+\nu\right)\right)-1\right)^{2}} & \text { при } \beta<0, \\
\frac{\beta G \exp \left(-\beta\left(\lambda_{n}+\nu\right)\right)}{\left(\exp \left(-\beta\left(\lambda_{n}+\nu\right)\right)-1\right)^{2}} & \text { при } \beta>0 .\end{cases}
\end{aligned}
$$


В силу (95), во-первых, уравнение (25) имеет решение $\nu>-\lambda_{1}$ для любых $\beta<0$ и $\nu<-\lambda_{n}$ для любых $\beta>0$; обозначим это решение через $\nu(\beta, N)$. Во-вторых, из $(93)$ и (95) следует, что

$$
\nu^{\prime}=\nu(\beta, N)+O\left(\frac{1}{N^{1 / 4}}\right)
$$

так как для $D \tilde{N}(\beta, \nu)(78)$ согласно $(10)$ имеет место оценка

$$
\begin{aligned}
& \frac{-\beta G \exp \left(-\beta\left(\lambda_{n}+\nu\right)\right)}{\left(\exp \left(-\beta\left(\lambda_{n}+\nu\right)\right)-1\right)^{2}} \leqslant D \tilde{N}(\beta, \nu) \leqslant \frac{-\beta G \exp \left(-\beta\left(\lambda_{1}+\nu\right)\right)}{\left(\exp \left(-\beta\left(\lambda_{1}+\nu\right)\right)-1\right)^{2}} \quad \text { при } \beta<0, \\
& \frac{\beta G \exp \left(-\beta\left(\lambda_{1}+\nu\right)\right)}{\left(\exp \left(-\beta\left(\lambda_{1}+\nu\right)\right)-1\right)^{2}} \leqslant D \tilde{N}(\beta, \nu) \leqslant \frac{\beta G \exp \left(-\beta\left(\lambda_{n}+\nu\right)\right)}{\left(\exp \left(-\beta\left(\lambda_{n}+\nu\right)\right)-1\right)^{2}} \quad \text { при } \beta>0,
\end{aligned}
$$

а $G$ растет как $N$ по условию (21). Учтем теперь, что для $\widetilde{N}_{\alpha}(\beta, \nu)$ вьполняется (79) и они, следовательно, обладают свойствами, аналогичными (95). Тог да из (96), (79), (95) и определения $\mathscr{N}_{\alpha}(\beta, N)(24)$ следует, что

$$
\left|\mathscr{N}_{\alpha}(\beta, N)-\widetilde{N}_{\alpha}\left(\beta, \nu^{\prime}\right)\right|=O\left(N^{3 / 4}\right) .
$$

Пусть теперь $\Delta=a N^{3 / 4+\delta}$, где $a$ и $\delta$ - произвольные положительные параметры, не зависящие от $N$. Если вьполнено условие

$$
\left|\tilde{N}_{\alpha}\left(\beta, \nu^{\prime}\right)-N_{\alpha}\right| \geqslant \Delta
$$

то при достаточно больших $N$ вьполнено условие

$$
\sum_{\alpha=1}^{m}\left|\mathscr{N}_{\alpha}(\beta, N)-N_{\alpha}\right| \geqslant \Delta^{\prime}=\Delta-\sum_{\alpha=1}^{m}\left|\mathcal{N}_{\alpha}(\beta, N)-\tilde{N}_{\alpha}\left(\beta, \nu^{\prime}\right)\right|
$$

Отсюда следует неравенство

$$
\begin{aligned}
& \sum_{\{k\}} \Theta\left(\sum_{\alpha=1}^{m}\left|N_{\alpha}-\mathcal{N}_{\alpha}(\beta, \nu)\right|-\Delta\right) \gamma(\{k\}) \exp (\beta(\mathscr{B}(\{k\})+\nu N(\{k\}))) \\
& \quad \leqslant \sum_{\{k\}} \Theta\left(\sum_{\alpha=1}^{m}\left|N_{\alpha}-\tilde{N}_{\alpha}\left(\beta, \nu^{\prime}\right)\right|-\Delta^{\prime}\right) \gamma(\{k\}) \exp (\beta(\mathscr{B}(\{k\})+\nu N(\{k\}))) .
\end{aligned}
$$

В силу (98) и (43) для любого не зависящего от $N$ параметра $\varepsilon>0$ и для всех положительных $a$ и $\delta$ при $N \rightarrow \infty$ справедлива оценка

$$
\sqrt{N} \exp \left(-\frac{\Delta^{\prime 2}}{2 G d}\right)=O\left(\exp \left(-\frac{(1-\varepsilon) a^{2} N^{1 / 2+2 \delta}}{2 \widetilde{g} d}\right)\right) .
$$

Из (85), (94) и (100) следует (27). Теорема доказана.

ЗАмЕчАнИЕ. Здесь рассмотрен случай $\beta>0$. Совершенно аналогично рассматривается случай $\beta<0$. Если везде брать $\nu<-\alpha_{n}$, то можно получить те же результаты. 
5. Туннельный канонический оператор в экономике. Равновесныецены определяются из условия равенства спроса и предложения по каждому продукту и ресурсу. Аналогично определяются следующие пары: потоки продуктов и услуг - цены; потоки труда разных видов - ставки зарплаты; потоки природных ресурсов - ренты; процент ссуды - объем ссуд.

Асимптотика $M$ и $\widetilde{M}$ дается туннельным каноническим оператором в фазовом пространстве пар [5].

Мы будем рассматривать фазовое пространство $\mathbb{R}^{2 n}$, где интенсивные величины будут играть роль координат, а экстенсивные - импульсов. В экономике роль значений случайной величины $\lambda_{i}$ могут играть цены соответствующих товаров, а $N_{i}$ - например, число проданных, т.е. число людей, купивших данный товар, или процент, выплачиваемьй $i$-м банком и т.д. Очевидно, что цена зависит от спроса, т.е. $\lambda_{i}\left(N_{i}\right)$ в двухмерном фазовом пространстве - это кривая. В двухмерном фазовом пространстве каждой точке (вектору) $\lambda_{i}, i=1, \ldots, n$, отвечает некоторьй вектор $N_{i}\left(\lambda_{1}, \ldots, \lambda_{n}\right), i=1, \ldots, n$. В более общем случае это $N$-мерное многообразие (поверхность), где "координаты" и "импульсы" локально зависят от $n$ параметров; при этом выполняется некоторое условие: скобки Лагранжа от “координат" и “импульсов" по этим параметрам равны нулю. Поэтому автор назвал такое многообразие лагранжевьг. По-другому можно сказать, что форма $\sum N_{i} d \lambda_{i}$ замкнута (см. послесловие [6] и [7]). Значит, $\int N_{i} d \lambda_{i}$ не зависит от пути и, как в механике $\int p d q(p$ - импульс, $q$ - координата), назьвается действием.

Производитель приобретает ресурсы и преобразовьвает вектор затрат ресурсов в вектор вьпусков потребительских благ. Потребитель приобретает эти блага. Соответственно определяются вышеизложенньми равенствами равновесные цены на ресурсы и цены на потребительские блага [8]-[17].

Кроме таких равновесных цен могут еще образовываться вертикальные пары на отдельные потребительские блага и пары продавец - покупатель, т.е. постоянный продавец - постоянньй покупатель, и соответственно образовываются цены, связанные с этими парами. Аналогом в квантовой статистике являются куперовские пары.

Эта конструкция требует привлечение метода ультравторичного квантования в абстрактной алгебраической форме, которая могла бы быть применима в экономике. Эта теория приводит и к образованию “вертикальных" кластеров. Этот метод разработан в другой работе.

Автор выражает глубокую благодарность Г. В. Ковалю за помощь и В. Н. Батурину за поддержку и обсуждение.

\section{СПИСОК ЦИТИРОВАННОЙ ЛИТЕРАТУРЫ}

[1] Колмогоров А. Н. // Избранные труды по математике и механике, 1985. С. 136-137.

[2] Маслов В. П. Аксиомы нелинейного осреднения в финансовой математике и динамика курса акций // Теор. вероятн. и ее примен. 2003. Т. 48. № 4. С. 800-810.

[3] 'Zero Intelligence' Trading Closely Mimics Stock Market // http://www .news cientist.com/ article.ns?id=dn6948. Katharine Davis: NewScientist, 05/02/01; см. также другие сайты по ссылке "Zero Intelligence".

[4] Вьюгин В. В., Маслов В. П. Теорема о концентрации для энтропии и свободной энергии // Проблемы передачи информации. 2005. Т. 41. № 2. С. 72-88.

[5] Маслов В. П. Геометрическое "квантование" термодинамики и статистические поправки в критических точках // ТМФ. 1994. Т. 101. № 3. С. 433-441. 
[6] Хединг Дж. Введение в метод фазового интервала // Послесловие к книге: Маслов В.П. Метод ВКБ в многомерном случае. М.: Мир, 1965.

[7] Маслов В. П. Теория возмущений и асимптотические методы. М.: МГУ, 1965.

[8] Маслов В. П. Аппроксимационные вероятности, закон квазистабильного рынка и фазовый переход из "конденсатного" состояния // Докл. РАН. 2003. Т. 392. №6. С. 727-732.

[9] Маслов В.П. Интегральные уравнения и фазовые переходы в вероятностных играх. Аналогия со статистической физикой // Теор. вероятн. и ее примен. 2003. Т. 48. № 2. С. 482-502.

[10] Маслов В. П. Эконофизика и квантовая статистика // Матем. заметки. 2002. Т. 72. №6. C. $883-891$.

[11] Маслов В. П. Зависимость покупательной способности и среднего дохода населения от числа покупателей на специализировнном рынке и в регионе. Законы эконофизики // Докл. РАН. 2004. T. 395. № 2. С. 164-168.

[12] Маслов В. П. Расходы покупателей и скорость оборота при нелинейном финансовом осреднении. Законы эконофизики // Докл. РАН. 2004. Т. 396. № 2.

[13] Маслов В. П. Нелинейное финансовое осреднение, эволюционный процесс и законы эконофизики // Теор. вероятн. и ее применен. 2004. Т. 49. № 2. С. 34.

[14] Маслов В. П. Квазистабильная экономика и ее связь с термодинамикой сверхтекучей жидкости. Дефолт как фазовый переход нулевого рода. 1 // Обозрение прикладной и промышленной математики. 2004. Т. 11. № 4. С. 690-732; 2 // Обозрение прикладной и промышленной математики. 2005. Т. 12. № 1. С. 3-40.

[15] Батурин В.Н., Лебедев С. Г., Маслов В.П., Садовников Б. И., Чеботарев А. М. Реконструкция Парето-распределения в области высоких доходов // Экономическая наука современной России. 2005. № 3 .

[16] Maslov V. P. Quantum economics // Russian J. Math. Phys. 2005. V. 12. № 2. P. 219-231.

[17] Маслов В.П. Капиталистическая математика // Manuscript at www.viktor-maslov. narod.ru, 2005.

Московский государственный университет им. М.В. Ломоносова

Поступило

E-mail : victor_maslov@hotmail.com 